\title{
Fabrication of miniaturized fluidic devices using SU-8 based lithography and low temperature wafer bonding
}

\author{
P. Svasek $^{\mathrm{a}, *}$, E. Svasek $^{\mathrm{a}}$, B. Lendl ${ }^{\mathrm{b}}$, M. Vellekoop ${ }^{\mathrm{c}}$ \\ a Ludwig Boltzmann Institute of Biomedical Microtechnology, Gusshausstrasse 27-29, A-1040 Vienna, Austria \\ ${ }^{\mathrm{b}}$ Institute of Chemical Technologies and Analytics, Vienna University of Technology, Vienna, Austria \\ ${ }^{\mathrm{c}}$ Institute of Sensor-and Actuatorsystems, Vienna University of Technology, Vienna, Austria \\ Received 22 September 2003; received in revised form 4 March 2004; accepted 13 March 2004 \\ Available online 19 May 2004
}

\begin{abstract}
In this paper, we present a technology for the batch-fabrication of fluidic devices which combines polymer and metal layers. The structures are fabricated by means of two-layer lithography and SU-8-based wafer bonding technique. The combination of SU-8 and metal layers allows the fabrication of "2(1/2)"-dimensional fluidic structures. We realized different types of micromixers for the investigation of chemical reactions by time resolved FTIR-spectroscopy, a flow-through-cell for IR-detection in capillary electrophoresis (CE) and a CE device with integrated capillary.
\end{abstract}

(C) 2004 Elsevier B.V. All rights reserved.

Keywords: SU-8; Micromixer; Fluidic device; FTIR-spectroscopy; Wafer bonding

\section{Introduction}

During the last years the negative-working photoresist SU-8 has become a widely used material for the fabrication of MEMS and miniaturized fluidic devices [1,2]. This epoxy-based material offers very good properties, such as high mechanical strenght, good adhesion on many different substrate materials and superior chemical stability. It can be processed with standard lithography equipment like spincoater and UV maskaligner. The achievable structure height is in the range from $<1$ to $1000 \mu \mathrm{m}$. Structures with high aspect ratio and high quality of the sidewalls can be produced.

Processing of SU-8 includes the following steps:

a. Deposition of the resist by spin- or spraycoating.

b. Drying (softbake) at $95^{\circ} \mathrm{C}$, preferably on a hotplate with a very flat surface.

c. Exposure with a standard UV maskaligner.

d. Post exposure bake at $90^{\circ} \mathrm{C}$ : exposed resist is crosslinked.

e. Development: unexposed resist is dissolved by a suitable solvent (propylene glycol monomethyl ether acetate, PGMEA)

\footnotetext{
* Corresponding author. Tel.: +43-1-58801-36643; fax: +43-1-58801-36699.

E-mail address: peter.svasek@tuwien.ac.at (P. Svasek).
}

f. Hardbake: the remaining structures are completely crosslinked. The resist gets its final mechanical strenght and chemical stability.

\section{Flow cell for infrared detection in capillary electrophoresis (CE) systems}

The fused silica capillaries normally used in capillary electrophoresis are not transparent to IR. Therefore, an infrared transparent flow-through cell had to be designed in order to use FTIR-spectroscopy as a detection method in CE-systems [3]. An appropriate material for this purpose is calcium fluoride $\left(\mathrm{CaF}_{2}\right)$. It shows high transmittance in the mid-IR-range [4].

\subsection{Fabrication of the CE-cell}

The cell consists of two plates of $\mathrm{CaF}_{2}$, each $1 \mathrm{~mm}$ thick (see Fig. 1). On one of these plates there is a titanium layer which acts as an optical aperture. This is necessary because the given optical setup cannot provide a focus smaller than the width of the flow channel. The $200 \mathrm{~nm}$ thick titanium layer is deposited by evaporation and patterned by conventional lift-off technique. This metal structure must be completely isolated from the flow channel, otherwise gas bubbles would be produced by electrolysis. 


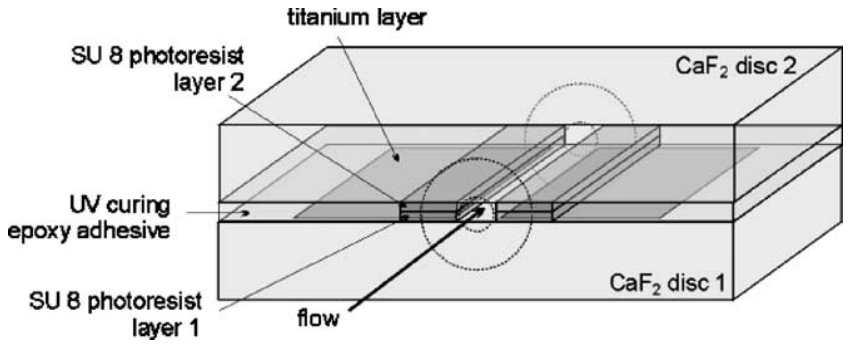

Fig. 1. Schematic drawing of the CE-cell. The size of the cell is $2 \mathrm{~mm} \times 5 \mathrm{~mm} \times 2 \mathrm{~mm}$. The IR-beam passes the flow channel in vertical direction.

The channel is formed by two lines of $\mathrm{SU}-8$ on each $\mathrm{CaF}_{2}$ plate, each $100 \mu \mathrm{m}$ wide. The distance between these two lines and consequently the width of the channel is $150 \mu \mathrm{m}$. The height of the SU-8 lines is $7.5 \mu \mathrm{m}$, half of the desired height of the cell. The $\mathrm{CaF}_{2}$ wafers (diameter $30 \mathrm{~mm}$, thickness $1 \mathrm{~mm}$ ) are spin-coated with SU-8 and softbaked. After UV-exposure using a SUSS MJB3 maskaligner and an appropriate photomask, the resist is post-exposure-baked $\left(90^{\circ} \mathrm{C}\right.$ for $10 \mathrm{~min}$ ) and developed. The unexposed and therefore not crosslinked areas are dissolved during the development process. The developer used is PGMEA.

Two wafers (one with and one without metal structure) are superimposed, aligned and pressed against each other during the hardbake process. Consequently a bond is established between corresponding SU-8 structures because the hardbake temperature of $150-200{ }^{\circ} \mathrm{C}$ induces the complete crosslinking of the SU-8. This hardbake is necessary to achieve the good mechanical and chemical properties of the resist. Improvement of this simple wafer bonding method is discussed later.

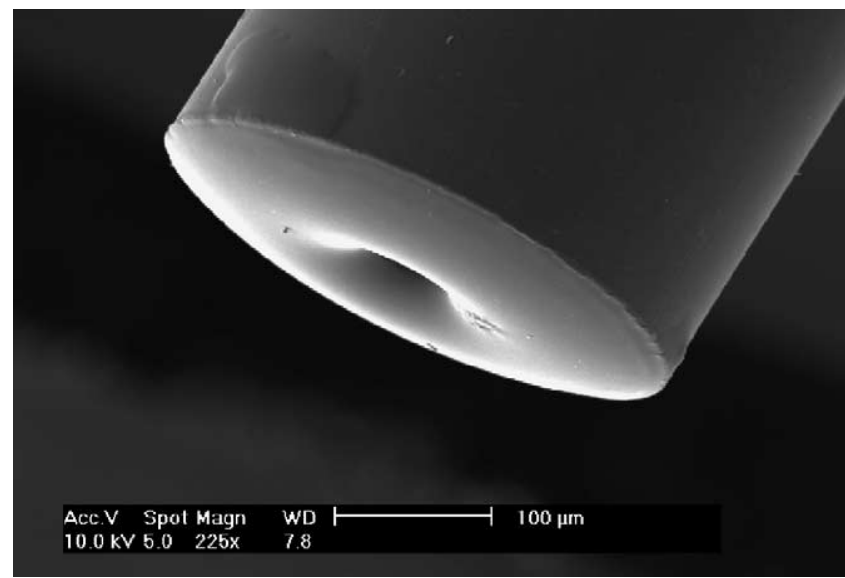

Fig. 2. Tip of a capillary with applied gasket. The soft epoxy material allows a tight connection which is virtually free of dead volume.

After hardbake of the SU-8 the area outside the SU-8 lines (i.e. outside the flow channels) is filled with UV curing epoxy adhesive (DELO Katiobond ${ }^{\circledR}$ 4653) and finally the waferstack is diced. We get 40 devices from one waferstack with a diameter of $30 \mathrm{~mm}$. The final dimension of the cell is $2 \mathrm{~mm} \times 5 \mathrm{~mm} \times 2 \mathrm{~mm}$.

\subsection{Connection between capillaries and the CE-cell}

To achieve a tight connection which is virtually free of dead volume a special setup is used. A gasket is applied to the tip of each capillary (see Fig. 2). This gasket consists of UV curing epoxy-material (DELO Katiobond ${ }^{\circledR} 4653$ ) which is rather soft (shore D 54) after curing. Blockage of the capillaries is prevented by a stream of air which passes the

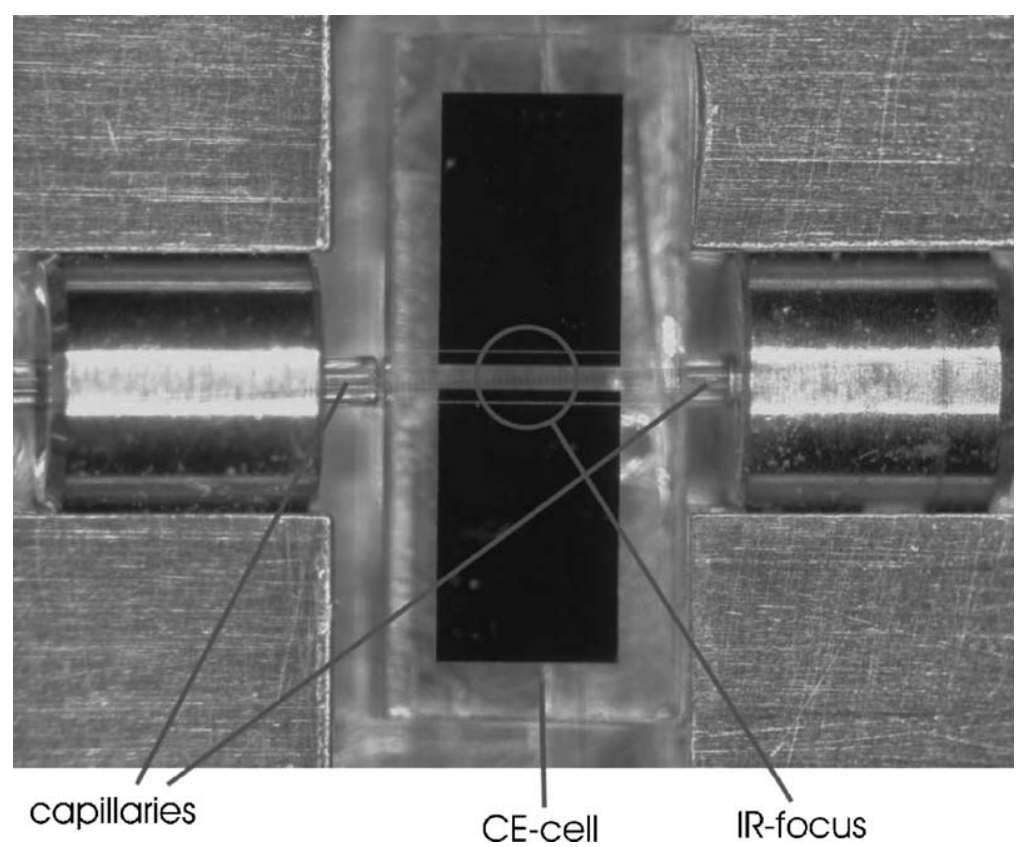

Fig. 3. CE-cell with attached capillaries. The capillaries are guided by bushes to ensure their correct position. 
capillaries during application and curing of the epoxy material. The outer diameter of the capillaries is $360 \mu \mathrm{m}$, the inner diameter is $50 \mu \mathrm{m}$. Two capillaries are coupled to the CE-cell by means of a brass holder. The cell is glued to the holder and the capillaries are pressed against the sidewalls of the cell and held by clamps. Their correct position with respect to the cell is ensured by two bushes which guide the capillaries when they are inserted (see Fig. 3). The two surfaces of each cell where the capillaries are coupled (inlet and outlet of the flow channel) are polished with $3 \mu \mathrm{m}$ lapping film to ensure a tight connection between the capillaries and the cell.

\subsection{Results}

To demonstrate that the CE-IR-cell does not influence the separation, experiments were carried out which included UV- and FTIR-detection in one setup. The results of an MEKC (micellar electrokinetic chromatography) are shown in Figs. 4 and 5.

The conditions were as follows:

Capillary: $50 \mu \mathrm{m}$ i.d., uncoated fused silica, $80 \mathrm{~cm}$ length (40 cm capillary/CE-FTIR-cell/20 $\mathrm{cm}$ capillary/UV detector $/ 20 \mathrm{~cm}$ capillary).

Buffer: $20 \mathrm{mM}$ phosphate, $40 \mathrm{mM}$ SDS, pH7, adjusted with $\mathrm{NaOH}$.

Separation voltage: $+20 \mathrm{kV}(18 \mu \mathrm{A})$ at injection end.

UV detection: $210 \mathrm{~nm}$.

Fig. 4 shows the result of the UV-detection. A very clear separation of five substances (paracetamol, Caffeine, $p$-nitrobenzylalkohol, $m$-nitrophenol, $p$-nitrophenol) has been obtained. This proves the suitability of the CE-FTIR-cell which has been fabricated with SU-8 technology.

The result of the FTIR-detection is shown in Fig. 5. The plot shows a series of IR-spectra which illustrates clearly

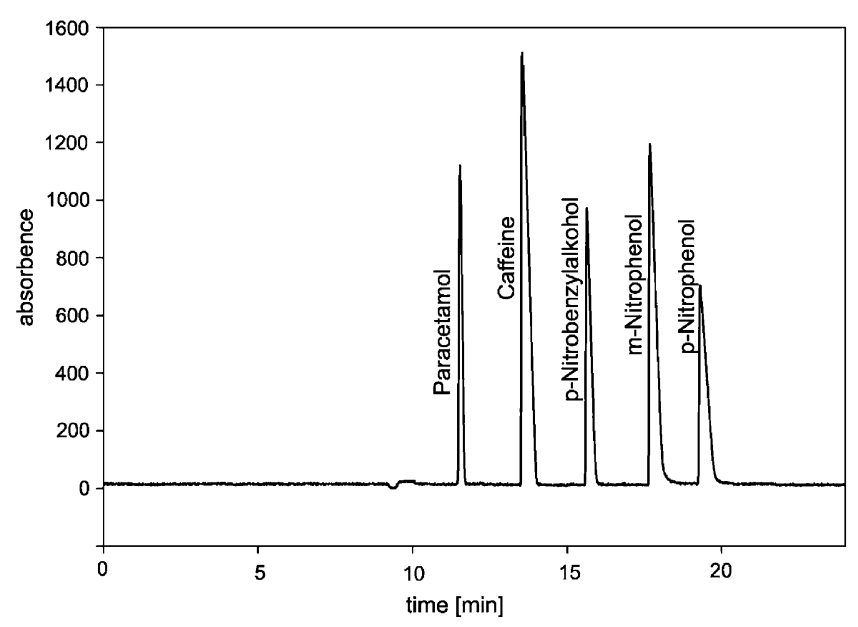

Fig. 4. Result of the UV-detection in an MEKC-separation with simultaneous UV- and FTIR-detection.

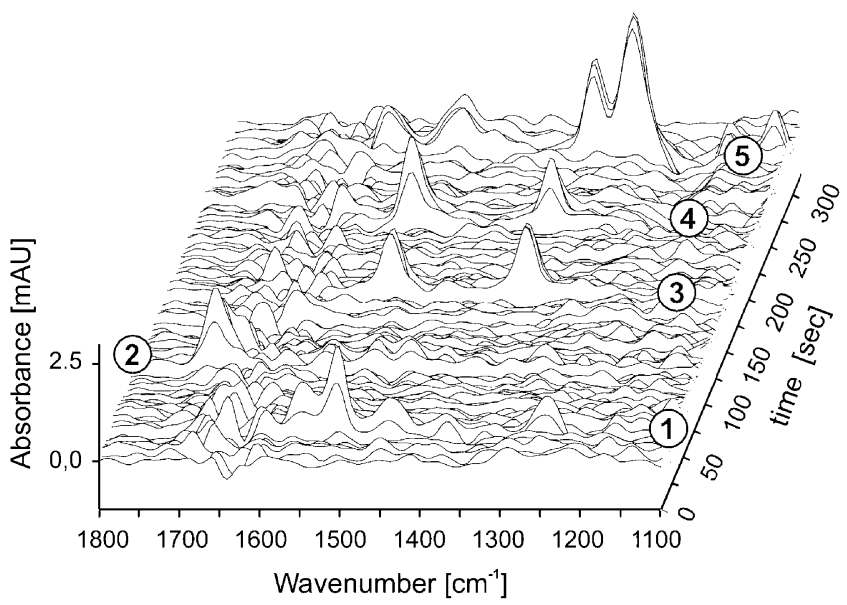

Fig. 5. Result of the FTIR-detection in an MEKC-separation with simultaneous UV-and FTIR-detection. The substances are: (1) paracetamol, (2) caffeine, (3) $p$-nitrobenzylalcohol, (4) $m$-nitrophenol, (5) $p$-nitrophenol.

that both detection and identification of the substances can be performed simultaneously.

Other results obtained from experiments that were carried out with the help of the CE-FTIR-setup are presented elsewhere $[2,4,5]$.

\section{Micromixer for time resolved FTIR-spectroscopy}

This mixing device is used for the investigation of chemical reactions by time resolved FTIR-spectroscopy combined with in situ mixing of two reactants $[4,6,7]$.

\subsection{Working principle of the micromixer}

The device is Y-shaped (see Fig. 6). The two liquids injected through the two inlet ports are separated by the separation membrane until they meet inside the mixing chamber. This membrane is a $2 \mu \mathrm{m}$ thick silver membrane. Two thin sheets of liquid are superimposed inside the mixing chamber and mixed by vertical diffusion. The mixer is operated in the "stopped flow" mode, i.e. the two liquids are injected by means of a double syringe pump. During the injection there is hardly any premixing. Then the flow is stopped, the two reactants are mixed by diffusion and the chemical reaction takes place. This reaction is investigated by time resolved FTIR-spectroskopy [7]. The stop of the flow is the "trigger" for the start of the reaction.

Because of the cross-section of the mixing chamber, which is $1 \mathrm{~mm}$ wide and $10 \mu \mathrm{m}$ high, the time needed for complete mixing is rather short. Fluid dynamic simulations performed with FLUENT V5.5 showed as a result a mixing time of $100 \mathrm{~ms}$ for aqueous solutions and based on a height of the mixing chamber of $20 \mu \mathrm{m}$ [4]. The height of the latest devices is $10 \mu \mathrm{m}$ so one can assume a mixing time of approx. $25 \mathrm{~ms}$. 


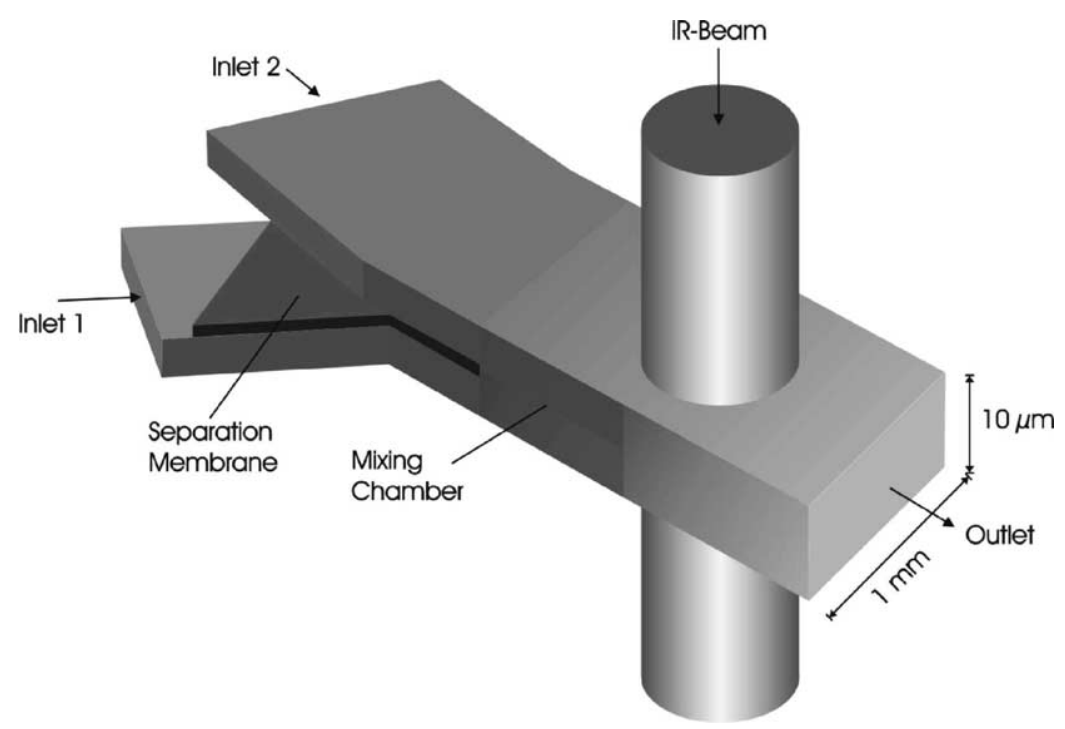

Fig. 6. Working principle of the micromixer.

The IR-beam directly passes the mixing chamber. The optical pathlength (the height of the mixing chamber) is $10 \mu \mathrm{m}$ (see Fig. 6). This pathlength should not exceed $20 \mu \mathrm{m}$ because of the strong absorption of water and organic solvents in the mid-IR region. A pathlength in excess of approximately $20 \mu \mathrm{m}$ would result in a poor signal-to-noise-ratio.

\subsection{Fabrication of the micromixer}

The micromixer is fabricated by means of a two-layer lithography, which combines SU-8 and metal, together with a polymer-based wafer bonding technique. As mentioned above, calcium fluoride is used as substrate material. The wafers which are used have a diameter of $100 \mathrm{~mm}$, a thickness of $1 \mathrm{~mm}$ and are double side polished. A $4 \mu \mathrm{m}$ thick layer of SU-8 is deposited by spin-coating onto both wafers. To achieve this layer thickness, the SU-8-50, which has a rather high viscosity, has to be diluted 4:1 (v/v) with GBL (gamma-butyro-lactone). Spin coating is done at $5000 \mathrm{rpm}$. After spin-coating the wafers are softbaked at $90^{\circ} \mathrm{C}$ for 30 min. Exposure of wafer \#1 is done by means of a SUSS MA 150 mask aligner. Wafer \#1 is the bottom wafer. It carries the structures for inlet 1 and the separation membrane (see Fig. 6). Exposure is followed by a post-exposure bake at $90^{\circ} \mathrm{C}$ for $10 \mathrm{~min}$. A hotplate is used for all baking processes except the wafer bonding process.

To form the separation membrane a $2 \mu \mathrm{m}$ thick metal layer is deposited by evaporation on top of the exposed (but undeveloped) SU-8 layer. We choose Ag as a material for this layer for two reasons: evaporated $\mathrm{Ag}$ layers show no internal stress, and therefore, they do not bend when released. Additionally, it is possible to deposit thick Ag layers by thermal evaporation with relatively low power at a temperature of the evaporation source well below $1000^{\circ} \mathrm{C}$. Consequently no UV light is generated, which would expose the wafer during the evaporation process, because the SU-8 layer is still sensitive to light. Evaporation is done in a Balzers BAK 550 evaporation system. The Ag layer is then covered by positive photoresist AZ 1512 HS. This photoresist is processed as usual and the Ag-layer is wet etched to form the structure of the separation membrane. As an etchant an aqueous $45 \%$ (WT) solution of $\mathrm{Fe}\left(\mathrm{NO}_{3}\right)_{3}$ is used. This solution does not attack the SU-8. The photomask for the Ag layer is compensated for the underetch of the Ag.

Finally the AZ photoresist is removed by undiluted developer AZ $400 \mathrm{k}$ and the SU-8 structure is developed with PGMEA. To dissolve unexposed SU-8 underneath the metal structure takes quite some time (approx. $2 \mathrm{~h}$ for channels of $1 \mathrm{~mm}$ length). Wafer \#1 now carrys the structures of inlet 1 and the separation membrane. The narrow SU-8-walls above and beneath the separation membrane are necessary to guide the flow and to keep the Ag-membrane in place (see Fig. 7). On wafer \#2 (the top wafer) the structure of inlet 2 is fabricated as usual from a single $4 \mu \mathrm{m}$ thick layer of SU-8. Subsequently the holes for two inlets and one outlet per mixer (see Fig. 8) are drilled by means of a high speed spindle and diamond tools. So far the SU-8 structures on both wafers are not hardbaked and therefore the polymer is still able to establish a bond to other materials during hardbake.

To complete the production process of the micromixer both wafers need to be bonded together in order to form a closed fluidic structure.

\section{Adhesive wafer bonding with $\mathrm{SU}-8$}

It is reported that a bonding strenght of $\sim 20 \mathrm{MPa}$ can be achieved if SU-8 is used as intermediate layer for adhesive wafer bonding [8]. Wafer bonding with SU-8 can be performed simply by applying contact force and heat to a stack of wafers which carry SU-8 structures [9]. Both wafers are superimposed to form a sandwich-configuration, 


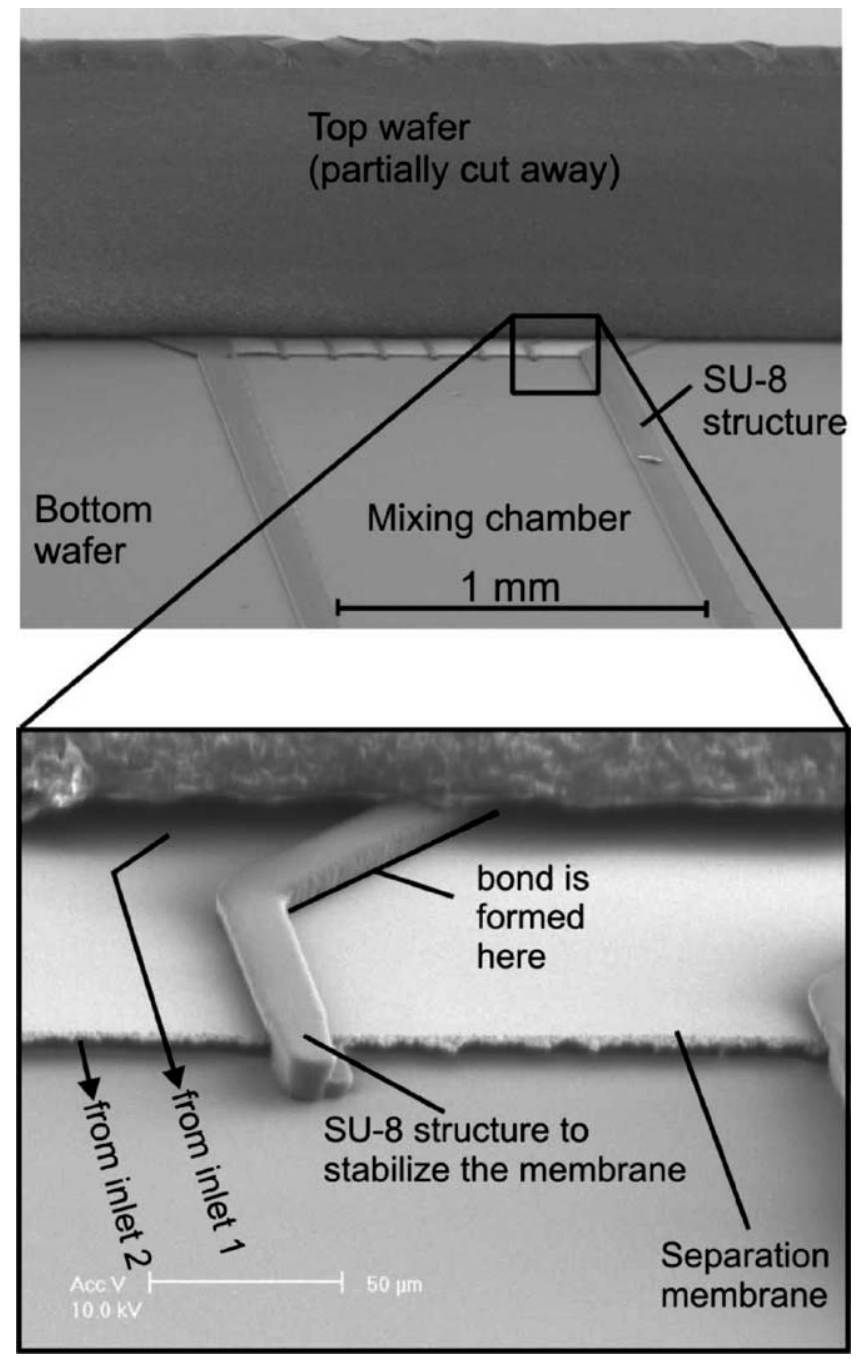

Fig. 7. SEM-micrograph of the micromixer. The top wafer is partially cut away to allow a look into the mixing chamber.

and aligned. The bonding process is done in an EVG 501 wafer bonder. The wafers are pressed against each other with a contact force of $2000 \mathrm{~N}$ and heated to $150^{\circ} \mathrm{C}$ for $1 \mathrm{~h}$. Because of this high temperature the SU- 8 is hardbaked and a

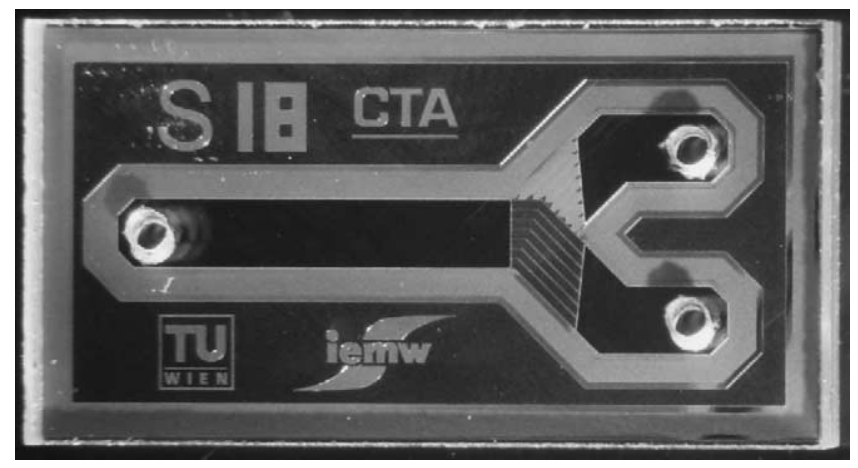

Fig. 8. Micrograph of the mixing device. The size of the device is $6.2 \mathrm{~mm} \times 12.7 \mathrm{~mm} \times 2 \mathrm{~mm}$.

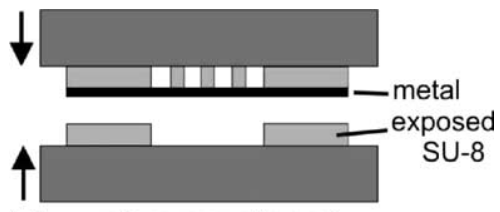

(a) wafers are aligned

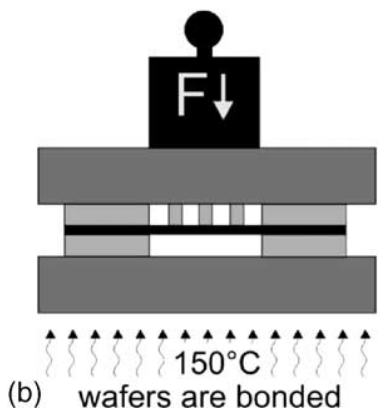

Fig. 9. Wafer bonding process without the use of unexposed SU-8.

bond is established between the SU-8 structure on the top wafer and the SU-8-Ag structure on the bottom wafer (see Fig. 9).

A drawback of this bonding method results from the fact that the layer thickness uniformity of spin-coated SU-8 layers is limited. For a layer thickness of approximately $4 \mu \mathrm{m}$ we were able to achieve thickness differences of $0.05 \mu \mathrm{m}$ throughout the surface of a 4 in. wafer. The thickness of the layers was measured after development of the structures using a DEKTAK 8 stylus profiler. Due to these differences the corresponding structures are not entirely in close contact. Consequently some voids remain after bonding [10], which decreases the yield of the bonding process. Our bonding experiments showed a yield of approximately $30 \%$, i.e. 20 devices of 62 showed perfect bonding.

One way to overcome this problem is to increase the contact force. SU-8-structures which are not hardbaked are plastic and can be deformed. Consequently some gaps or voids can be closed if a higher contact force is applied. The required force depends on the contact area as well as on the aspect ratio of the structures to be bonded. For this reason this method is feasable for the CE-cell because here the size of the wafers is only $30 \mathrm{~mm}$ and if there are some gaps after bonding, these gaps are sealed by the epoxy adhesive (see Section 2). On the other hand, if the structures are deformed too much, the desired shape and height of the fluidic structures cannot be maintained and will vary throughout the wafer area.

A new method to avoid insufficient bonding is to add some soft "adhesive" which fills the gaps caused by non-uniform layer thickness. Again the material of choice is SU-8, but this time without UV exposure. Softbaked and unexposed SU-8 is thermoplastic, and at approx. $90^{\circ} \mathrm{C}$ it even flows and fills gaps because of capillary forces. When heated to $150-200^{\circ} \mathrm{C}$ SU-8 is crosslinked, even without UV exposure. One can say that it behaves like thermally curing epoxy adhesive. A 

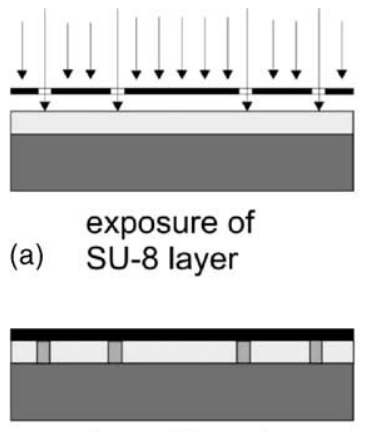

deposition of

(c) metal layer

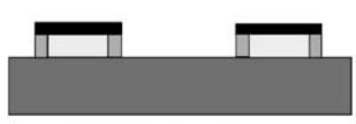

SU-8 structure

(e) developed

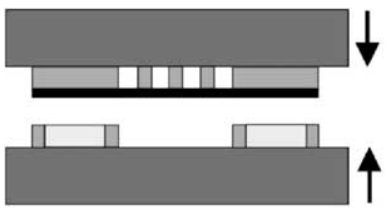

wafers are aligned

(g)

Fig. 10. Improved wafer bonding process. Steps (a-f) show the preparation of one of the wafers. Steps ( $g$ and $h$ ) show the actual bonding. layer of unexposed SU-8 can be used as an adhesive layer for waferbonding [10]. If SU-8 is used for building fluid structures and-in unexposed form-for waferbonding, the unexposed SU-8 has to be patterned. This method is applied as follows (see Fig. 10).

After exposure and PEB a metal layer is deposited on top of the SU-8 layer by evaporation. This metal layer $(1 \mu \mathrm{m}$ $\mathrm{Ag}$ ) is patterned by wet etching in the same way as described in Section 3. Now the areas where unexposed SU-8 is desired are surrounded by narrow walls of exposed SU-8 and covered by metal. After development of the SU- 8 structure and removal of the metal layer the wafer is ready for bonding. Alignment of the wafers is done using an EVG AL6 mask aligner. Subsequently two wafers are clamped to the bondtool, but still separated. The bondtool is inserted into an EVG 501 wafer bonder. The chamber of the wafer bonder is evacuated and the wafers are brought into contact. Then the temperature of the top and bottom heaters is ramped up to $150{ }^{\circ} \mathrm{C}$ with $3{ }^{\circ} \mathrm{C} / \mathrm{min}$. This temperature is maintained for $1 \mathrm{~h}$ and finally ramped down to room temperature. Because of the high thermal expansion $(17 \mathrm{ppm} / \mathrm{K})$ and the very high brittleness of calcium fluoride wafers, uniform heat distribution as well as slow temperature ramps are very important to avoid breakage of wafers caused by stress.

\subsection{Results}

If this improved bonding method is used, it is not necessary to apply a very high contact force, because remaining gaps are closed by the unexposed (soft) SU-8 and consequently the bonding yield is increased to approximately $90 \%$, which was investigated by optical inspection (see Fig. 8).

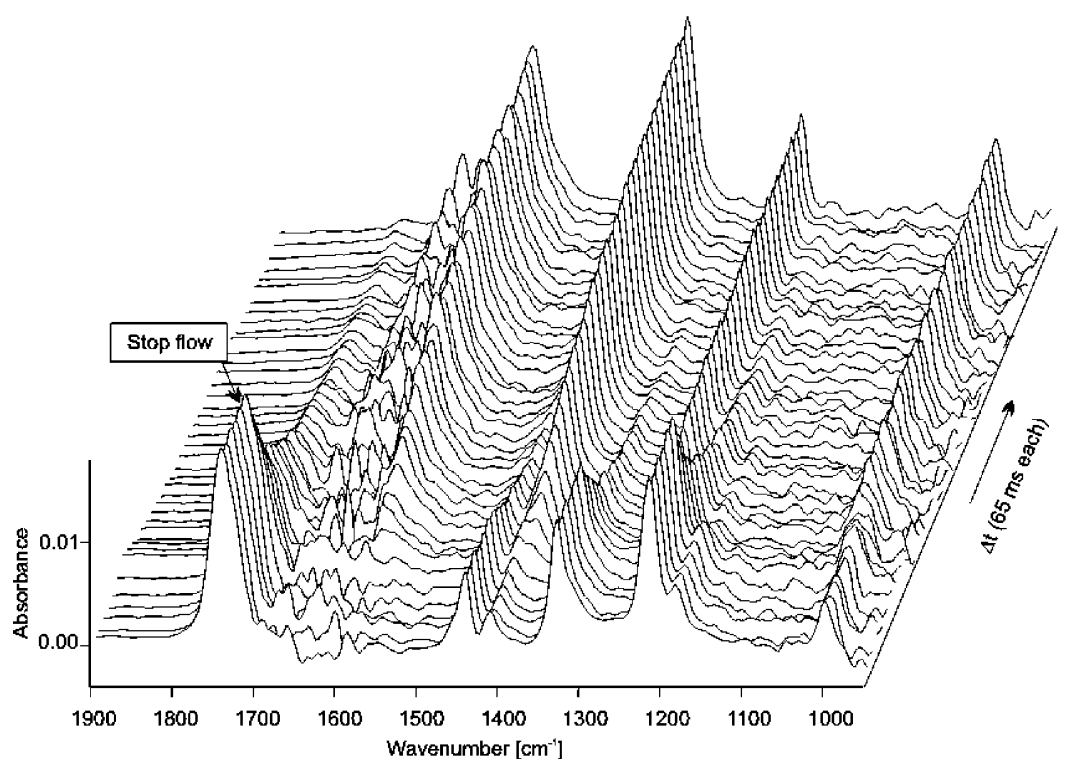

Fig. 11. Stack plot of FTIR spectra obtained from a time resolved run of the reaction of methyl monochloroacetate and sodium hydroxide. Time delay between subsequent spectra is $65 \mathrm{~ms}$. 


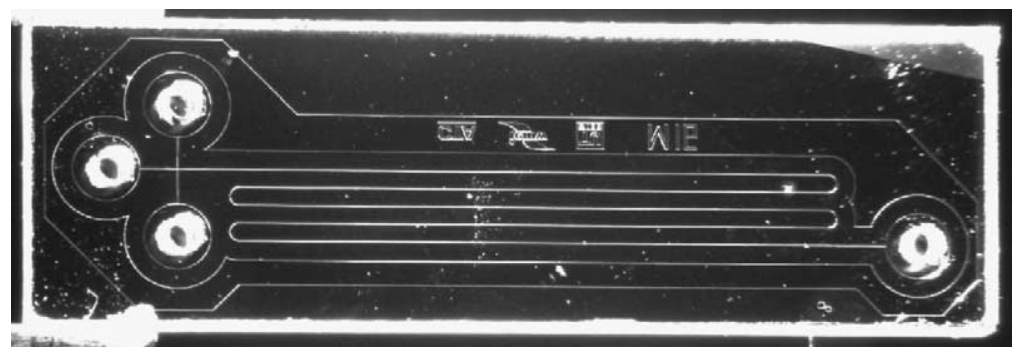

Fig. 12. Triple mixer with narrow mixing channel for measurements in the "continous-flow-mode". The size of the device is $5.1 \mathrm{~mm} \times 16.3 \mathrm{~mm} \times 2 \mathrm{~mm}$.

As an example, Fig. 11 shows a three-dimensional plot of a series of IR-spectra obtained from an experiment using the described device. The reaction is the saponification of methyl monochloroacetate with sodium hydroxide. No formation of the reaction products is observed during the flow on. After stopping of the flow the spectrum of the reaction products can be observed. Because of the fact that the mixing time is in the range of only $25 \mathrm{~ms}$ (if the mixing channel is $10 \mu \mathrm{m}$ high), one can monitor very clearly the progress of the chemical reaction as shown in Fig. 11. Additionally, very fast reactions can be used to test the performance of the mixer [4].

\section{Triple mixer and integrated CE-device}

Recently developed devices are a triple mixer and an integrated CE-device. These devices are currently under test. Both devices are intended to be used for time resolved FTIR-spectroscopy as well, but in this case with an IR-microscope attached to a synchrotron source. The advantage of this setup is the possibility to have a very small measuring spot (approx. $10 \mu \mathrm{m}$ ), and consequently the fluid channels can be made very narrow. Compared to the previously described mixer both devices are simple structures - they consist of a single channel in one plane. The triple mixer has three input ports and one output port. Mixing occures by lateral diffusion. The mixing channel is $15 \mu \mathrm{m}$ wide, $10 \mu \mathrm{m}$ high, approximately $50 \mathrm{~mm}$ long and meander-shaped (see Fig. 12). This mixer has been used in the "continuous-flow" mode. Two or three reactants are continuously pumped through the device and the IR-spectra are measured at different locations along the mixing channel. If the mixing time is short compared to the time which is necessary for complete passage of the channel, different phases of the chemical reaction can be "seen" while the IR-focus is moved along the channel. Another possibility is to use a fixed measuring spot while the flow rate is varied. By applying this "continuous-flow" mode one can avoid the problems with the fluidic setup for the "stopped-flow" mode. For a reliable measurement, a very complicated setup is required to achieve a highly synchronous and very well defined stop of two liquids in the microfluidic system.

The integrated CE-device has three input ports for the injection of buffer and sample, one output port and a linear channel which is $150 \mu \mathrm{m}$ wide and $10 \mu \mathrm{m}$ high. The length of the channel is $40 \mathrm{~mm}$. The dimension of the device is $44.1 \mathrm{~mm} \times 4.4 \mathrm{~mm} \times 2 \mathrm{~mm}$. For chemical reasons-the channel should be surrounded by one kind of material only-a thin $(0.5 \mu \mathrm{m})$ layer of SU-8 was deposited on both wafers prior to the fabrication of the structures. These thin layers do not considerably decrease the IR-transmission.

Because of the fact that the triple mixer and the CE-device are fabricated using the same technology, both kinds of device are located on the same wafer. As for the micromixers described in this paper, $1 \mathrm{~mm}$ thick, 4 in. $\mathrm{CaF}_{2}$ wafers are used and the same improved wafer bonding method which was described above, was applied.

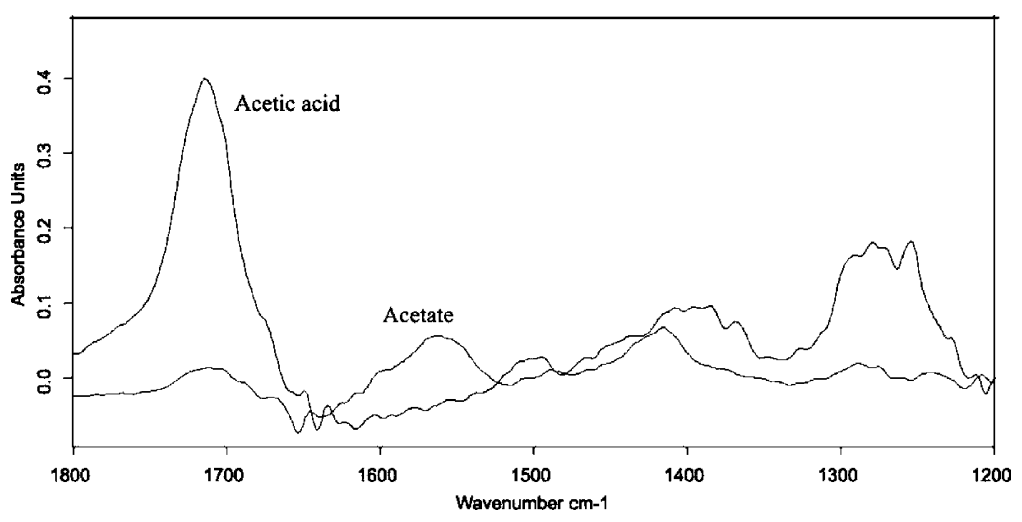

Fig. 13. Preliminary result of an experiment with the triple mixer. The spectrum of Acetic acid is replaced by the spectrum of Acetate when NaOH is added. 


\subsection{Preliminary results}

The above-described triple mixer has been recently tested using synchrotron radiation at the installations of ANKA and BESSY II. BESSY beamline provides a very intense radiation that is in addition quite homogeneously distributed along the spectral range of interest. Therefore, according to our experience, the beam provided by BESSY II is more suitable for mid-IR experiments.

Some preliminary results using the described micromixer have already been obtained at the FTIR beamline at BESSY. Fig. 13 shows the FTIR spectra measured for $0.5 \mathrm{M}$ acetic acid introduced into the mixer through the central channel while the outer channels are filled with water. When water is replaced by $0.25 \mathrm{M} \mathrm{NaOH}$, the acid-base reaction takes place and the spectrum of acetate ion can be observed, proving then the mixing of the reagents. Additionally, no leakages or blockages could be observed. However, from these experiments it was established that the geometrical design of the device causes some problems for optical measurements and has to be modified. Therefore, an improved version of the micromixer will be developed to enable further biological measurements.

For the integrated CE-device the detection of a single substance (myoglobin) could be proven during a preliminary experiment using an IR-microscope attached to a synchrotron source.

\section{Conclusion}

The SU-8 based fabrication method described in this paper is suitable for the batch fabrication of microfluidic devices. The combination of the epoxy-based photoresist SU-8 and metal allows to build up structures which contain fluid channels and chambers in more than one plane. SU-8 can be used not only for the definition of the fluidic structures, but for waferbonding as well. If hard and soft (i.e. exposed and unexposed) SU-8 is used in combination, this wafer bonding method is a possibility to overcome the problem of non-uniform thickness of the SU-8 layers. High bonding strength can be achieved with this low-temperature bonding process. Additionally, this method allows the bonding of wafers which contain conducting lines or other structures which cause a topographic surface. Several different microfluidic devices fabricated with this new method have shown leak-free and stable operation.

\section{Acknowledgements}

This work was financially supported by the Ludwig Boltzmann Institute of Biomedical Microtechnology, Vienna. All described devices have been tested at the Institute of Chemical Technology and Analytics, Vienna University of Technology.

\section{References}

[1] H. Lorenz, M. Despont, M. Fahrni, N. LaBianca, P. Vettiger, P. Renaud, SU-8: a low cost negative resist for MEMS, J. Micromech. Microeng. 7 (1997) 121-124.

[2] L. Guerin, M. Bossel, M. Demierre, S. Calmes, P. Renaud, Simple and low cost fabrication of embedded microchannels by using a new thick-film photoplastic, in: Proceedings on Transducers 1997, Chicago, 1997, pp. 1419-1422.

[3] M. Kölhed, P. Hinsmann, P. Svasek, J. Frank, B. Karlberg, B. Lendl, On-line fourier transform infrared detection in capillary electrophoresis, Anal. Chem. 74 (2002) 3843-3848.

[4] P. Hinsmann, J. Frank, P. Svasek, M. Harasek, B. Lendl, Design, simulation and application of a new micromixing device for time resolved infrared spectroscopy of chemical reactions in solution, Lab. Chip 1 (2001) 16-21.

[5] B. Lendl, M. Kölhed, P. Hinsmann, M. Haberkorn, P. Svasek, B. Karlberg, On-line mid-IR (quantum cascade laser and FTIR spectrometric) detection in capillary based separation systems, in: Proceedings of the $\mu$ TAS 2002 Symposium, Nara, Japan, 2002, ISBN: 1-4020-1009-5 599-601.

[6] B. Lendl, P. Hinsmann, P. Svasek, J. Frank, Device for label-free bio-ligand interaction studies based on time resolved fourier transform infrared spectrometry, in: Proceedings of the $\mu$ TAS 2002 Symposium, 2002, ISBN: 1-420-1009-5 221-223.

[7] P. Hinsmann, M. Haberkorn, J. Frank, P. Svasek, M. Harasek, B. Lendl, Time-resolved FTIR spectroscopy of chemical reactions in solution by fast diffusion-based mixing in a micromachined flow cell, Appl. Spectrosc. 55 (3) (2001) 241-251.

[8] C.T. Pan, H. Yang, S.C. Shen, M.C. Chou, H.P. Chou, A low-temperature wafer bonding technique using patternable materials, J. Micromech. Microeng. 12 (2002) 611-615.

[9] S.K. Sampath, L.St. Clair, X. Wu, D.V. Ivanov, G. Wang, C. Ghosh, K.R. Farmer, Rapid MEMS prototyping using SU-8, wafer bonding and deep reactive ion etching, in: Proceedings of the 14 , Biennial-University/Government/Industry, Microelectronics Symposium Cat. No. 01CH37197, 2001, pp. 158-161.

[10] S. Li, C.B. Freidhoff, R.M. Young, R. Ghodssi, Fabrication of micronozzles using low-temperature wafer-level bonding with SU-8, J. Micromech. Microeng. 13 (2003) 732-738.

\section{Biographies}

Peter Svasek was born in Vienna 1951. He attended a technical college (HTL) in Vienna and received his diploma in electronic engineering 1977. From 1977 to 1984 he worked in the field of industrial electronics. Since 1984, he has been working at the Institute of Sensor- and Actuatorsystems of the Vienna University of Technology. The main topics of his work are instrumentation for miniaturised electrochemical sensors and biosensors, thin film technology, microfluidics and low temperature wafer bonding.

Edeltraud Svasek was born in Vienna in 1951. She attended a college of Electronic Engineering from 1989 to 1994. Since 1986 she has been working as a technician at the Vienna University of Technology at the Institute of Sensor- and Actuator Systems in the field of thinfilm based sensortechnology.

Bernhard Lendl studied chemistry at the Vienna University of Technology 1987-1993. After doing his diploma work at the Department of Analytical Chemistry in Córdoba (Spain) he received his master of science in 1993. He then joined the group of professor R. Kellner for his $\mathrm{PhD}$, focusing on novel flow analysis based applications of Fourier transform infrared spectroscopy. After finishing his habilitation in 2001, he was appointed associate professor for analytical chemistry 
at the Institute of Chemical Technologies and Analytics of the Vienna University of Technology. So far he is author or co-author of over 70 scientific papers and holds two international patents. His current research interests centre around novel instrumental developments based on vibrational spectroscopic detectors and their application in Analytical Chemistry.

Michael J. Vellekoop was born in Amsterdam in 1960. He received the BSc degree in physics in 1982 and the $\mathrm{PhD}$ degree in electrical engineering in 1994. In 1988, he co-founded Xensor Integration B.V. where he was managing director until 1996. In that year, he initiated a new group on the topic of physical chemosensors at the DIMES Electronic Instrumentation Laboratory of the Delft University of Technology, where in 1997 he became an associated professor. Since 2001 he is a full professor of industrial sensor systems at the Institute of Sensor and Actuator Systems at the Vienna University of Technology, Austria. Keywords of research are physical chemosensors, sensor systems, lab on a chip, micro and nanofluidics, micro and nanotechnology. He authored and co-authored more than 130 publications in peer reviewed journals and international conferences. 